FACTA UNIVERSITATIS (NIŠ)

Ser. Math. Inform. Vol. 35, No 5 (2020), 1291-1304

https://doi.org/10.22190/FUMI2005291G

\title{
ON PSEUDO-HERMITIAN MAGNETIC CURVES IN SASAKIAN MANIFOLDS
}

\author{
Şaban Güvenç and Cihan Özgür
}

(c) 2020 by University of Niš, Serbia | Creative Commons Licence: CC BY-NC-ND

Abstract. We define pseudo-Hermitian magnetic curves in Sasakian manifolds endowed with the Tanaka-Webster connection. After we have given a complete classification theorem, we shall construct parametrizations of pseudo-Hermitian magnetic curves in $\mathbb{R}^{2 n+1}(-3)$.

Keywords: magnetic curve; slant curve; Sasakian manifold; the Tanaka-Webster connection.

\section{Introduction}

The study of the motion of a charged particle in a constant and time-independent static magnetic field on a Riemannian surface is known as the Landau-Hall problem [16]. The main problem is to study the movement of a charged particle moving in the Euclidean plane $\mathbb{E}^{2}$. The solution of the Lorentz equation (called also the Newton equation) corresponds to the motion of the particle. The trajectory of a charged particle moving on a Riemannian manifold under the action of the magnetic field is a very interesting problem from a geometric point of view [16].

Let $(N, g)$ be a Riemannian manifold, and $F$ a closed 2-form, $\Phi$ the Lorentz force, which is a $(1,1)$-type tensor field on $N . F$ is called a magnetic field if it is associated to $\Phi$ by the relation

$$
F(X, Y)=g(\Phi X, Y),
$$

where $X$ and $Y$ are vector fields on $N$ (see [1], [3] and [8]). Let $\nabla$ be the Riemannian connection on $N$ and consider a differentiable curve $\alpha: I \rightarrow N$, where $I$ denotes an open interval of $\mathbb{R}$. $\alpha$ is said to be a magnetic curve for the magnetic field $F$, if it is a solution of the Lorentz equation given by

$$
\nabla_{\alpha^{\prime}(t)} \alpha^{\prime}(t)=\Phi\left(\alpha^{\prime}(t)\right) .
$$

Received February 28, 2020; accepted April 04, 2020

2020 Mathematics Subject Classification. Primary 53C25; Secondary 53C40, 53A04 
From the definition of magnetic curves, it is straightforward to see that their speed is constant. Specifically, unit-speed magnetic curves are called normal magnetic curves [9].

In [9], Druţă-Romaniuc, Inoguchi, Munteanu and Nistor studied magnetic curves in a Sasakian manifold. Magnetic curves in cosymplectic manifolds were studied in [10] by the same authors. In [13], 3-dimensional Berger spheres and their magnetic curves were considered by Inoguchi and Munteanu. Magnetic trajectories of an almost contact metric manifold were studied in [14], by Jleli, Munteanu and Nistor. The classification of all uniform magnetic trajectories of a charged particle moving on a surface under the action of a uniform magnetic field was obtained in [19], by Munteanu. Furthermore, normal magnetic curves in para-Kaehler manifolds were researched in [15], by Jleli and Munteanu. In [17], Munteanu and Nistor obtained the complete classification of unit-speed Killing magnetic curves in $\mathbb{S}^{2} \times \mathbb{R}$. Moreover, in [18], they studied magnetic curves on $\mathbb{S}^{2 n+1}$. 3-dimensional normal para-contact metric manifolds and their magnetic curves of a Killing vector field were investigated in [5], by Calvaruso, Munteanu and Perrone. In [20], the present authors studied slant curves in contact Riemannian 3-manifolds with pseudo-Hermitian proper mean curvature vector field and pseudo-Hermitian harmonic mean curvature vector field for the Tanaka-Webster connection in the tangent and normal bundles, respectively. The second author gave the parametric equations of all normal magnetic curves in the 3-dimensional Heisenberg group in [21]. Recently, the present authors have also considered slant magnetic curves in $S$-manifolds in [11].

These studies motivate us to investigate pseudo-Hermitian magnetic curves in $(2 n+1)$-dimensional Sasakian manifolds endowed with the Tanaka-Webster connection. In Section 2, we summarize the fundamental definitions and properties of Sasakian manifolds and the unique connection, namely the Tanaka-Webster connection. We give the main classification theorems for pseudo-Hermitian magnetic curves in Section 3. We show that a pseudo-Hermitian magnetic curve cannot have osculating order greater than 3. In the last section, after a brief information on $\mathbb{R}^{2 n+1}(-3)$, we obtain the parametric equations of pseudo-Hermitian magnetic curves in $\mathbb{R}^{2 n+1}(-3)$ endowed with the Tanaka-Webster connection.

\section{Preliminaries}

Let $N$ be a $(2 n+1)$-dimensional Riemannian manifold satisfying the following equations

$$
\begin{gathered}
\phi^{2}(X)=-X+\eta(X) \xi, \quad \eta(\xi)=1, \quad \phi(\xi)=0, \quad \eta \circ \phi=0, \\
g(X, \xi)=\eta(X), \quad g(X, Y)=g(\phi X, \phi Y)+\eta(X) \eta(Y),
\end{gathered}
$$

for all vector fields $X, Y$ on $N$, where $\phi$ is a $(1,1)$-type tensor field, $\eta$ is a 1-form, $\xi$ is a vector field and $g$ is a Riemannian metric on $N$. In this case, $(N, \phi, \xi, \eta, g)$ is said to be an almost contact metric manifold [2]. Moreover, if $d \eta(X, Y)=\Phi(X, Y)$, 
where $\Phi(X, Y)=g(X, \phi Y)$ is the fundamental 2-form of the manifold, then $N$ is said to be a contact metric manifold [2].

Furthermore, if we denote the Nijenhuis torsion of $\phi$ by $[\phi, \phi]$, for all $X, Y$ $\in \chi(N)$, the condition given by

$$
[\phi, \phi](X, Y)=-2 d \eta(X, Y) \xi
$$

is called the normality condition of the almost contact metric structure. An almost contact metric manifold turns into a Sasakian manifold if the normality condition is satisfied [2].

From Lie differentiation operator in the characteristic direction $\xi$, the operator $h$ is defined by

$$
h=\frac{1}{2} L_{\xi} \phi
$$

It is directly found that the structural operator $h$ is symmetric. It also validates the equations below, where we denote the Levi-Civita connection by $\nabla$ :

$$
h \xi=0, \quad h \phi=-\phi h, \quad \nabla_{X} \xi=-\phi X-\phi h X,
$$

(see [2]).

If we denote the Tanaka-Webster connection on $N$ by $\widehat{\nabla}([22],[24])$, then we have

$$
\widehat{\nabla}_{X} Y=\nabla_{X} Y+\eta(X) \phi Y+\left(\widehat{\nabla}_{X} \eta\right)(Y) \xi-\eta(Y) \nabla_{X} \xi
$$

for all vector fields $X, Y$ on $N$. By the use of equations (2.3), the Tanaka-Webster connection can be calculated as

$$
\widehat{\nabla}_{X} Y=\nabla_{X} Y+\eta(X) \phi Y+\eta(Y)(\phi X+\phi h X)-g(\phi X+\phi h X, Y) \xi .
$$

The torsion of the Tanaka-Webster connection is

$$
\widehat{T}(X, Y)=2 g(X, \phi Y) \xi+\eta(Y) \phi h X-\eta(X) \phi h Y .
$$

In a Sasakian manifold, from the fact that $h=0$ (see [2]), the equations (2.4) and (2.5) can be rewritten as:

$$
\begin{gathered}
\widehat{\nabla}_{X} Y=\nabla_{X} Y+\eta(X) \phi Y+\eta(Y) \phi X-g(\phi X, Y) \xi, \\
\widehat{T}(X, Y)=2 g(X, \phi Y) \xi .
\end{gathered}
$$

The following proposition states why the Tanaka-Webster connection is unique:

Proposition 2.1. [23] The Tanaka-Webster connection on a contact Riemannian manifold $N=(N, \phi, \xi, \eta, g)$ is the unique linear connection satisfying the following four conditions:
(a) $\widehat{\nabla} \eta=0, \widehat{\nabla} \xi=0$;
(b) $\widehat{\nabla} g=0, \widehat{\nabla} \phi=0$;
(c) $\widehat{T}(X, Y)=-\eta([X, Y]) \xi, \quad \forall X, Y \in D$;
(d) $\widehat{T}(\xi, \phi Y)=-\phi \widehat{T}(\xi, Y), \quad \forall Y \in D$. 


\section{Magnetic Curves with respect to the Tanaka-Webster Connection}

Let $(N, \phi, \xi, \eta, g)$ be an $n$-dimensional Riemannian manifold and $\alpha: I \rightarrow N$ a curve parametrized by arc-length. If there exists $g$-orthonormal vector fields $E_{1}, E_{2}, \ldots, E_{r}$ along $\alpha$ such that

$$
\begin{aligned}
E_{1} & =\alpha^{\prime}, \\
\widehat{\nabla}_{E_{1}} E_{1} & =\widehat{k}_{1} E_{2}, \\
\widehat{\nabla}_{E_{1}} E_{2} & =-\widehat{k}_{1} E_{1}+\widehat{k}_{2} E_{3}, \\
& \cdots \\
\widehat{\nabla}_{E_{1}} E_{r} & =-\widehat{k}_{r-1} E_{r-1},
\end{aligned}
$$

then $\alpha$ is called a Frenet curve for $\widehat{\nabla}$ of osculating order $r,(1 \leq r \leq n)$. Here $\widehat{k}_{1}, \ldots, \widehat{k}_{r-1}$ are called pseudo-Hermitian curvature functions of $\alpha$ and these functions are positive valued on I. A geodesic for $\widehat{\nabla}$ (or pseudo-Hermitian geodesic) is a Frenet curve of osculating order 1 for $\widehat{\nabla}$. If $r=2$ and $\widehat{k}_{1}$ is a constant, then $\alpha$ is called a pseudo-Hermitian circle. A pseudo-Hermitian helix of order $r(r \geq 3)$ is a Frenet curve for $\widehat{\nabla}$ of osculating order $r$ with non-zero positive constant pseudoHermitian curvatures $\widehat{k}_{1}, \ldots, \widehat{k}_{r-1}$. If we shortly state pseudo-Hermitian helix, we mean its osculating order is $3[7]$.

Let $N=\left(N^{2 n+1}, \phi, \xi, \eta, g\right)$ be a Sasakian manifold endowed with the TanakaWebster connection $\widehat{\nabla}$. Let us denote the fundamental 2 -form of $N$ by $\Omega$. Then, we have

$$
\Omega(X, Y)=g(X, \phi Y),
$$

(see [2]). From the fact that $N$ is a Sasakian manifold, we have $\Omega=d \eta$. Hence, $d \Omega=0$, i.e., it is closed. Thus, we can define a magnetic field $F_{q}$ on $N$ by

$$
F_{q}(X, Y)=q \Omega(X, Y),
$$

namely the contact magnetic field with strength $q$, where $X, Y \in \chi(N)$ and $q \in \mathbb{R}$ [14]. We will assume that $q \neq 0$ to avoid the absence of the strength of magnetic field (see [4] and [9]).

From (1.1) and (3.2), the Lorentz force $\Phi$ associated to the contact magnetic field $F_{q}$ can be written as

$$
\Phi=-q \phi
$$

So the Lorentz equation (1.2) is

$$
\nabla_{E_{1}} E_{1}=-q \phi E_{1},
$$

where $\alpha: I \rightarrow N$ is a curve with arc-length parameter, $E_{1}=\alpha^{\prime}$ is the tangent vector field and $\nabla$ is the Levi-Civita connection (see [9] and [14]). By the use of equations (2.6) and (3.3), we have

$$
\widehat{\nabla}_{E_{1}} E_{1}=\left[-q+2 \eta\left(E_{1}\right)\right] \phi E_{1} .
$$


Definition 3.1. Let $\alpha: I \rightarrow N$ be a unit-speed curve in a Sasakian manifold $N=\left(N^{2 n+1}, \phi, \xi, \eta, g\right)$ endowed with the Tanaka-Webster connection $\widehat{\nabla}$. Then it is called a normal magnetic curve with respect to the Tanaka-Webster connection $\widehat{\nabla}$ (or shortly a pseudo-Hermitian magnetic curve) if it satisfies equation (3.4).

If $\eta\left(E_{1}\right)=\cos \theta$ is a constant, then $\alpha$ is called a slant curve [6]. From the definition of pseudo-Hermitian magnetic curves, we have the following direct result as in the Levi-Civita case:

Proposition 3.1. If $\alpha$ is a pseudo-Hermitian magnetic curve in a Sasakian manifold, then it is a slant curve.

Proof. Let $\alpha: I \rightarrow N$ be a pseudo-Hermitian magnetic curve. Then, we find

$$
\begin{aligned}
\frac{d}{d t} g\left(E_{1}, \xi\right) & =g\left(\widehat{\nabla}_{E_{1}} E_{1}, \xi\right)+g\left(E_{1}, \widehat{\nabla}_{E_{1}} \xi\right) \\
& =g\left(\left[-q+2 \eta\left(E_{1}\right)\right] \phi E_{1}, \xi\right) \\
& =0
\end{aligned}
$$

So we obtain

$$
\eta\left(E_{1}\right)=\cos \theta=\text { constant }
$$

which completes the proof.

As a result, we can rewrite equation (3.4) as

$$
\widehat{\nabla}_{E_{1}} E_{1}=(-q+2 \cos \theta) \phi E_{1},
$$

where $\theta$ is the contact angle of $\alpha$. Now, we can state the following theorem:

Theorem 3.1. Let $\left(N^{2 n+1}, \phi, \xi, \eta, g\right)$ be a Sasakian manifold endowed with the Tanaka-Webster connection $\widehat{\nabla}$. Then $\alpha: I \rightarrow N$ is a pseudo-Hermitian magnetic curve if and only if it belongs to the following list:

(a) pseudo-Hermitian non-Legendre slant geodesics (including pseudo-Hermitian geodesics as integral curves of $\xi$ );

(b) pseudo-Hermitian Legendre circles with $\widehat{k}_{1}=|q|$ and having the Frenet frame field (for $\widehat{\nabla}$ )

$$
\left\{E_{1},-\operatorname{sgn}(q) \phi E_{1}\right\}
$$

(c) pseudo-Hermitian slant helices with

$$
\widehat{k}_{1}=|-q+2 \cos \theta| \sin \theta, \widehat{k}_{2}=|-q+2 \cos \theta| \varepsilon \cos \theta
$$

and having the Frenet frame field (for $\widehat{\nabla}$ )

$$
\left\{E_{1}, \frac{\delta}{\sin \theta} \phi E_{1}, \frac{\varepsilon}{\sin \theta}\left(\xi-\cos \theta E_{1}\right)\right\},
$$

where $\delta=\operatorname{sgn}(-q+2 \cos \theta), \varepsilon=\operatorname{sgn}(\cos \theta)$ and $\cos \theta \neq \frac{q}{2}$. 
Proof. Let us assume that $\alpha: I \rightarrow N$ is a normal magnetic curve with respect to $\widehat{\nabla}$. Consequently, equation (3.5) must be validated. Let us assume $\widehat{k}_{1}=0$. Hence, we have $\cos \theta=\frac{q}{2}$ or $\phi E_{1}=0$. If $\cos \theta=\frac{q}{2}$, then $\alpha$ is a pseudo-Hermitian non-Legendre slant geodesic. Otherwise, $\phi E_{1}=0$ gives us $E_{1}= \pm \xi$. Thus, $\alpha$ is a pseudo-Hermitian geodesic as an integral curve of $\pm \xi$. So we have just proved that $\alpha$ belongs to (a) from the list, if the osculating order $r=1$. Now, let $\widehat{k}_{1} \neq 0$. From equation (3.5) and the Frenet equations for $\hat{\nabla}$, we find

$$
\widehat{\nabla}_{E_{1}} E_{1}=\widehat{k}_{1} E_{2}=(-q+2 \cos \theta) \phi E_{1} .
$$

Since $E_{1}$ is unit, the equation (2.2) gives us

$$
g\left(\phi E_{1}, \phi E_{1}\right)=\sin ^{2} \theta .
$$

By the use of (3.6) and (3.7), we obtain

$$
\widehat{k}_{1}=|-q+2 \cos \theta| \sin \theta,
$$

which is a constant. Let us denote $\delta=\operatorname{sgn}(-q+2 \cos \theta)$. From (3.8), we can write

$$
\phi E_{1}=\delta \sin \theta E_{2} .
$$

Let us assume $\widehat{k}_{2}=0$, that is, $r=2$. From the fact that $\widehat{k}_{1}$ is a constant, $\alpha$ is a pseudo-Hermitian circle. (3.9) gives us

$$
\eta\left(\phi E_{1}\right)=0=\delta \sin \theta \eta\left(E_{2}\right),
$$

which is equivalent to

$$
\eta\left(E_{2}\right)=0 .
$$

Differentiating this last equation with respect to $\widehat{\nabla}$, we obtain

$$
\widehat{\nabla}_{E_{1}} \eta\left(E_{2}\right)=0=g\left(\widehat{\nabla}_{E_{1}} E_{2}, \xi\right)+g\left(E_{2}, \widehat{\nabla}_{E_{1}} \xi\right) .
$$

Since $\widehat{\nabla} \xi=0$ and $r=2$, we have

$$
g\left(-\widehat{k}_{1} E_{1}, \xi\right)=0
$$

that is, $\eta\left(E_{1}\right)=0$. Hence, $\alpha$ is Legendre and $\cos \theta=0$. From equation (3.8), we get $\widehat{k}_{1}=|q|$. In this case, we also obtain $\delta=-\operatorname{sgn}(q)$ and $E_{2}=-\operatorname{sgn}(q) \phi E_{1}$. We have proved that $\alpha$ belongs to (b) from the list, if the osculating order $r=2$. Now, let us assume $\widehat{k}_{2} \neq 0$. If we use $\widehat{\nabla} \phi=0$, we calculate

$$
\widehat{\nabla}_{E_{1}} \phi E_{1}=\widehat{k}_{1} \phi E_{2} .
$$

From (2.1) and (3.9), we find

$$
\phi^{2} E_{1}=-E_{1}+\cos \theta \xi=\delta \sin \theta \phi E_{2},
$$


which gives us

$$
\phi E_{2}=\frac{\delta}{\sin \theta}\left(-E_{1}+\cos \theta \xi\right)
$$

So equation (3.10) becomes

$$
\widehat{\nabla}_{E_{1}} \phi E_{1}=\widehat{k}_{1} \frac{\delta}{\sin \theta}\left(-E_{1}+\cos \theta \xi\right) .
$$

If we differentiate the equation (3.9) with respect to $\widehat{\nabla}$, we also have

$$
\begin{aligned}
\widehat{\nabla}_{E_{1}} \phi E_{1} & =\delta \sin \theta \widehat{\nabla}_{E_{1}} E_{2} \\
& =\delta \sin \theta\left(-\widehat{k}_{1} E_{1}+\widehat{k}_{2} E_{3}\right) .
\end{aligned}
$$

By the use of (3.12) and (3.13), we obtain

$$
\widehat{k}_{1} \cot \theta\left(\xi-\cos \theta E_{1}\right)=\widehat{k}_{2} \sin \theta E_{3} .
$$

One can easily see that

$$
g\left(\xi-\cos \theta E_{1}, \xi-\cos \theta E_{1}\right)=\sin ^{2} \theta .
$$

From (3.14), we calculate

$$
\widehat{k}_{2}=|-q+2 \cos \theta| \varepsilon \cos \theta,
$$

where we denote $\varepsilon=\operatorname{sgn}(\cos \theta)$. As a result, we get

$$
\begin{gathered}
E_{3}=\frac{\varepsilon}{\sin \theta}\left(\xi-\cos \theta E_{1}\right), \\
E_{2}=\frac{\delta}{\sin \theta} \phi E_{1} .
\end{gathered}
$$

If we differentiate (3.15) with respect to $\widehat{\nabla}$, since $\phi E_{1} \| E_{2}$, we find $\widehat{k}_{3}=0$. So we have just completed the proof of (c). Considering the fact that $\widehat{k}_{3}=0$, the Gram-Schmidt process ends. Thus, the list is complete.

Conversely, let $\alpha: I \rightarrow N$ belong to the given list. It is easy to show that equation (3.5) is satisfied. Hence, $\alpha$ is a pseudo-Hermitian magnetic curve.

A pseudo-Hermitian geodesic is said to be a pseudo-Hermitian $\phi$-curve if the set $s p\left\{E_{1}, \phi E_{1}, \xi\right\}$ is $\phi$-invariant. A Frenet curve of osculating order $r=2$ is said to be a pseudo-Hermitian $\phi$-curve if $s p\left\{E_{1}, E_{2}, \xi\right\}$ is $\phi$-invariant. A Frenet curve of osculating order $r \geq 3$ is said to be a pseudo-Hermitian $\phi$-curve if $\operatorname{sp}\left\{E_{1}, E_{2}, \ldots, E_{r}\right\}$ is $\phi$-invariant.

Theorem 3.2. Let $\alpha: I \rightarrow N$ be a pseudo-Hermitian $\phi$-helix of order $r \leq 3$, where $N=\left(N^{2 n+1}, \phi, \xi, \eta, g\right)$ is a Sasakian manifold endowed with the Tanaka-Webster connection $\widehat{\nabla}$. Then: 
(a) If $\cos \theta= \pm 1$, then it is an integral curve of $\xi$, i.e. a pseudo-Hermitian geodesic and it is a pseudo-Hermitian magnetic curve for $F_{q}$ for arbitrary $q$;

(b) If $\cos \theta \notin\{-1,0,1\}$ and $\widehat{k}_{1}=0$, then it is a pseudo-Hermitian non-Legendre slant geodesic and it is a pseudo-Hermitian magnetic curve for $F_{2 \cos \theta}$;

(c) If $\cos \theta=0$ and $\widehat{k}_{1} \neq 0$, i.e. $\alpha$ is a Legendre $\phi$-curve, then it is a pseudoHermitian magnetic circle generated by $F_{-\delta \widehat{k}_{1}}$, where $\delta=\operatorname{sgn}\left(g\left(\phi E_{1}, E_{2}\right)\right)$;

(d) If $\cos \theta=\frac{\varepsilon \widehat{k}_{2}}{\sqrt{\widehat{k}_{1}^{2}+\widehat{k}_{2}^{2}}}$ and $\widehat{k}_{2} \neq 0$, then it is a pseudo-Hermitian magnetic curve

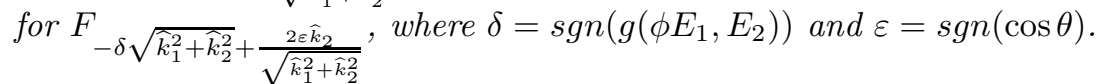

(e) Except above cases, $\alpha$ cannot be a pseudo-Hermitian magnetic curve for any $F_{q}$.

Proof. Firstly, let us assume $\cos \theta= \pm 1$, that is, $E_{1}= \pm \xi$. As a result, we have

$$
\widehat{\nabla}_{E_{1}} E_{1}=0, \phi E_{1}=0 \text {. }
$$

Hence, equation (3.5) is satisfied for arbitrary $q$. This proves (a). Now, let us take $\cos \theta \notin\{-1,0,1\}$ and $\widehat{k}_{1}=0$. In this case, we obtain

$$
\widehat{\nabla}_{E_{1}} E_{1}=0, \phi E_{1} \neq 0 \text {. }
$$

So equation (3.5) is valid for $q=2 \cos \theta$. The proof of (b) is over. Next, let us assume $\cos \theta=0$ and $\widehat{k}_{1} \neq 0$. One can easily see that $\alpha$ has the Frenet frame field (for $\widehat{\nabla}$ )

$$
\left\{E_{1}, \delta \phi E_{1}\right\}
$$

where $\delta$ corresponds to the sign of $g\left(\phi E_{1}, E_{2}\right)$. Consequently, we get

$$
\widehat{\nabla}_{E_{1}} E_{1}=\delta \widehat{k}_{1} \phi E_{1}
$$

that is, $\alpha$ is a pseudo-Hermitian magnetic curve for $q=-\delta \widehat{k}_{1}$. We have just proven (c). Finally, let $\cos \theta=\frac{\varepsilon \widehat{k}_{2}}{\sqrt{\widehat{k}_{1}^{2}+\widehat{k}_{2}^{2}}}$ and $\widehat{k}_{2} \neq 0$. So $\alpha$ has the Frenet frame field (for $\widehat{\nabla}$ )

$$
\left\{E_{1}, \frac{\delta}{\sin \theta} \phi E_{1}, \frac{\varepsilon}{\sin \theta}\left(\xi-\cos \theta E_{1}\right)\right\},
$$

where $\delta=\operatorname{sgn}\left(g\left(\phi E_{1}, E_{2}\right)\right)$ and $\varepsilon=\operatorname{sgn}(\cos \theta)$. After calculations, it is easy to show that equation (3.5) is satisfied for $q=-\delta \sqrt{\widehat{k}_{1}^{2}+\widehat{k}_{2}^{2}}+\frac{2 \varepsilon \widehat{k}_{2}}{\sqrt{\widehat{k}_{1}^{2}+\widehat{k}_{2}^{2}}}$. Hence, the proof of $(\mathrm{d})$ is completed. Except above cases, from Theorem 3.1, $\alpha$ cannot be a pseudo-Hermitian magnetic curve for any $F_{q}$. 


\section{Parametrizations of pseudo-Hermitian magnetic curves in $\mathbb{R}^{2 n+1}(-3)$}

In this section, our aim is to obtain parametrizations of pseudo-Hermitian magnetic curves in $\mathbb{R}^{2 n+1}(-3)$. To do this, we need to recall some notions from [2]. Let $N=\mathbb{R}^{2 n+1}$. Let us denote the coordinate functions of $N$ with $\left(x_{1}, \ldots, x_{n}, y_{1}, \ldots, y_{n}, z\right)$. One may define a structure on $N$ by $\eta=\frac{1}{2}\left(d z-\sum_{i=1}^{n} y_{i} d x_{i}\right)$, which is a contact structure, since $\eta \wedge(d \eta)^{n} \neq 0$. This contact structure has the characteristic vector field $\xi=2 \frac{\partial}{\partial z}$. Let us also consider a $(1,1)$-type tensor field $\phi$ given by the matrix form as

$$
\phi=\left[\begin{array}{ccc}
0 & \delta_{i j} & 0 \\
-\delta_{i j} & 0 & 0 \\
0 & y_{j} & 0
\end{array}\right]
$$

Finally, let us take the Riemannian metric on $N$ given by $g=\eta \otimes \eta+\frac{1}{4} \sum_{i=1}^{n}\left(\left(d x_{i}\right)^{2}+\right.$ $\left.\left(d y_{i}\right)^{2}\right)$. It is known that $(N, \phi, \xi, \eta, g)$ is a Sasakian space form and its $\phi$-sectional curvature is $c=-3$. This special Sasakian space form is denoted by $\mathbb{R}^{2 n+1}(-3)[2]$. One can easily show that the vector fields

$$
X_{i}=2 \frac{\partial}{\partial y_{i}}, X_{n+i}=\phi X_{i}=2\left(\frac{\partial}{\partial x_{i}}+y_{i} \frac{\partial}{\partial z}\right), i=\overline{1, n}, \xi=2 \frac{\partial}{\partial z}
$$

are $g$-unit and $g$-orthogonal. Hence, they form a $g$-orthonormal basis [2]. Using this basis, the Levi-Civita connection of $\mathbb{R}^{2 n+1}(-3)$ can be obtained as

$$
\begin{gathered}
\nabla_{X_{i}} X_{j}=\nabla_{X_{m+i}} X_{m+j}=0, \nabla_{X_{i}} X_{m+j}=\delta_{i j} \xi, \nabla_{X_{m+i}} X_{j}=-\delta_{i j} \xi \\
\nabla_{X_{i}} \xi=\nabla_{\xi} X_{i}=-X_{m+i}, \nabla_{X_{m+i}} \xi=\nabla_{\xi} X_{m+i}=X_{i}
\end{gathered}
$$

(see [2]). As a result, the Tanaka-Webster connection of $\mathbb{R}^{2 n+1}(-3)$ is

$$
\begin{aligned}
\widehat{\nabla}_{X_{i}} X_{j} & =\widehat{\nabla}_{X_{m+i}} X_{m+j}=\widehat{\nabla}_{X_{i}} X_{m+j}=\widehat{\nabla}_{X_{m+i}} X_{j}= \\
\widehat{\nabla}_{X_{i}} \xi & =\widehat{\nabla}_{\xi} X_{i}=\widehat{\nabla}_{X_{m+i}} \xi=\widehat{\nabla}_{\xi} X_{m+i}=0
\end{aligned}
$$

which was calculated in [12]. Now, we can investigate the parametric equations of pseudo-Hermitian magnetic curves in $\mathbb{R}^{2 n+1}(-3)$ endowed with the Tanaka-Webster connection.

Let $N=\mathbb{R}^{2 n+1}(-3)$ endowed with the Tanaka-Webster connection $\widehat{\nabla}$. Let $\alpha: I \subseteq \mathbb{R} \rightarrow N, \alpha=\left(\alpha_{1}, \alpha_{2}, \ldots, \alpha_{n}, \alpha_{n+1}, \ldots, \alpha_{2 n}, \alpha_{2 n+1}\right)$ be a pseudo-Hermitian magnetic curve. Then, the tangential vector field of $\alpha$ can be written as

$$
E_{1}=\sum_{i=1}^{n} \alpha_{i}^{\prime} \frac{\partial}{\partial x_{i}}+\sum_{i=1}^{n} \alpha_{n+i}^{\prime} \frac{\partial}{\partial y_{i}}+\alpha_{2 n+1}^{\prime} \frac{\partial}{\partial z}
$$

In terms of the $g$-orthonormal basis, $E_{1}$ is rewritten as

$$
E_{1}=\frac{1}{2}\left[\sum_{i=1}^{n} \alpha_{n+i}^{\prime} X_{i}+\sum_{i=1}^{n} \alpha_{i}^{\prime} X_{n+i}+\left(\alpha_{2 n+1}^{\prime}-\sum_{i=1}^{n} \alpha_{i}^{\prime} \alpha_{n+i}\right) \xi\right]
$$


From Proposition 3.1, $\alpha$ is a slant curve. Hence, we have

$$
\eta\left(E_{1}\right)=\cos \theta=\text { constant }
$$

which is equivalent to

$$
\alpha_{2 n+1}^{\prime}=2 \cos \theta+\sum_{i=1}^{n} \alpha_{i}^{\prime} \alpha_{n+i} .
$$

From the fact that $\alpha$ is parametrized by arc-length, we also have

$$
g\left(E_{1}, E_{1}\right)=1
$$

that is,

$$
\sum_{i=1}^{2 n}\left(\alpha_{i}^{\prime}\right)^{2}=4 \sin ^{2} \theta
$$

Differentiating $E_{1}$ with respect to $\widehat{\nabla}$, we obtain

$$
\widehat{\nabla}_{E_{1}} E_{1}=\frac{1}{2}\left(\sum_{i=1}^{n} \alpha_{n+i}^{\prime \prime} X_{i}+\sum_{i=1}^{n} \alpha_{i}^{\prime \prime} X_{n+i}\right) .
$$

We also easily find

$$
\phi E_{1}=\frac{1}{2}\left(-\sum_{i=1}^{n} \alpha_{i}^{\prime} X_{i}+\sum_{i=1}^{n} \alpha_{n+i}^{\prime} X_{n+i}\right) .
$$

Since $\alpha$ is a pseudo-Hermitian magnetic curve, it must satisfy

$$
\widehat{\nabla}_{E_{1}} E_{1}=(-q+2 \cos \theta) \phi E_{1} .
$$

Then, we can write

$$
\frac{\alpha_{n+1}^{\prime \prime}}{-\alpha_{1}^{\prime}}=\ldots=\frac{\alpha_{2 n}^{\prime \prime}}{-\alpha_{n}^{\prime}}=\frac{\alpha_{1}^{\prime \prime}}{\alpha_{n+1}^{\prime}}=\ldots=\frac{\alpha_{n}^{\prime \prime}}{\alpha_{2 n}^{\prime}}=-\lambda,
$$

where $\lambda=q-2 \cos \theta$. From the last equations, we can select the pairs

$$
\frac{\alpha_{n+1}^{\prime \prime}}{-\alpha_{1}^{\prime}}=\frac{\alpha_{1}^{\prime \prime}}{\alpha_{n+1}^{\prime}}, \ldots, \frac{\alpha_{2 n}^{\prime \prime}}{-\alpha_{n}^{\prime}}=\frac{\alpha_{n}^{\prime \prime}}{\alpha_{2 n}^{\prime}} .
$$

Firstly, let $\lambda \neq 0$. Solving the ODEs, we have

$$
\left(\alpha_{i}^{\prime}\right)^{2}+\left(\alpha_{n+i}^{\prime}\right)^{2}=c_{i}^{2}, i=1, \ldots, n
$$

for some arbitrary constants $c_{i}(i=1, \ldots, n)$ such that

$$
\sum_{i=1}^{n} c_{i}^{2}=4 \sin ^{2} \theta
$$


So we have

$$
\alpha_{i}^{\prime}=c_{i} \cos f_{i}, \alpha_{n+i}^{\prime}=c_{i} \sin f_{i}
$$

for some differentiable functions $f_{i}: I \rightarrow \mathbb{R}(i=1, \ldots, n)$. From (4.4), we get

$$
\frac{\alpha_{n+i}^{\prime \prime}}{-\alpha_{i}^{\prime}}=-f_{i}^{\prime}=-\lambda
$$

which gives us

$$
f_{i}=\lambda t+d_{i}
$$

for some arbitrary constants $d_{i}(i=1, \ldots, n)$. Here, $t$ denotes the arc-length parameter. Then, we find

$$
\alpha_{i}^{\prime}=c_{i} \cos \left(\lambda t+d_{i}\right), \alpha_{n+i}^{\prime}=c_{i} \sin \left(\lambda t+d_{i}\right)
$$

Finally, we obtain

$$
\begin{aligned}
& \alpha_{i}=\frac{c_{i}}{\lambda} \sin \left(\lambda t+d_{i}\right)+h_{i}, \\
& \alpha_{n+i}=\frac{-c_{i}}{\lambda} \cos \left(\lambda t+d_{i}\right)+h_{n+i}, \\
& \alpha_{2 n+1}=2 t \cos \theta+\sum_{i=1}^{n}\left\{\frac{-c_{i}^{2}}{4 \lambda^{2}}\left[2\left(\lambda t+d_{i}\right)+\sin \left(2\left(\lambda t+d_{i}\right)\right)\right]\right. \\
& \left.+\frac{c_{i} h_{n+i}}{\lambda} \sin \left(\lambda t+d_{i}\right)\right\}+h_{2 n+1}
\end{aligned}
$$

for some arbitrary constants $h_{i}(i=1, \ldots, 2 n+1)$.

Secondly, let $\lambda=0$. In this case, $q=2 \cos \theta$ and $\widehat{k}_{1}=0$. Hence, we have

$$
\widehat{\nabla}_{E_{1}} E_{1}=\frac{1}{2}\left(\sum_{i=1}^{n} \alpha_{n+i}^{\prime \prime} X_{i}+\sum_{i=1}^{n} \alpha_{i}^{\prime \prime} X_{n+i}\right)=0
$$

which gives us

$$
\begin{gathered}
\alpha_{i}=c_{i} t+d_{i}, i=1, \ldots, 2 n \\
\alpha_{2 n+1}=2 t \cos \theta+\sum_{i=1}^{n} c_{i}\left(\frac{c_{n+i}}{2} t^{2}+d_{n+i} t\right)+c_{2 n+1},
\end{gathered}
$$

where $c_{i}(i=1,2, \ldots, 2 n+1)$ and $d_{i}(i=1,2, \ldots, 2 n)$ are arbitrary constants such that

$$
\sum_{i=1}^{2 n} c_{i}^{2}=4 \sin ^{2} \theta
$$

To conclude, we can state the following theorem: 
Theorem 4.1. The pseudo-Hermitian magnetic curves on $\mathbb{R}^{2 n+1}(-3)$ endowed with the Tanaka-Webster connection have the parametric equations

$$
\alpha: I \subseteq \mathbb{R} \rightarrow \mathbb{R}^{2 n+1}(-3), \alpha=\left(\alpha_{1}, \alpha_{2}, \ldots, \alpha_{n}, \alpha_{n+1}, \ldots, \alpha_{2 n}, \alpha_{2 n+1}\right),
$$

where $\alpha_{i}(i=1, \ldots, 2 n+1)$ satisfies either

(a)

$$
\begin{gathered}
\alpha_{i}=\frac{c_{i}}{\lambda} \sin \left(\lambda t+d_{i}\right)+h_{i}, \\
\alpha_{n+i}=\frac{-c_{i}}{\lambda} \cos \left(\lambda t+d_{i}\right)+h_{n+i}, \\
\alpha_{2 n+1}=2 \cos \theta t+\sum_{i=1}^{n}\left\{\frac{-c_{i}^{2}}{4 \lambda^{2}}\left[2\left(\lambda t+d_{i}\right)+\sin \left(2\left(\lambda t+d_{i}\right)\right)\right]\right. \\
\left.+\frac{c_{i} h_{n+i}}{\lambda} \sin \left(\lambda t+d_{i}\right)\right\}+h_{2 n+1},
\end{gathered}
$$

where $\lambda=q-2 \cos \theta \neq 0, c_{i}, d_{i}(i=1, \ldots, n)$ and $h_{i}(i=1, \ldots, 2 n+1)$ are arbitrary constants such that

$$
\sum_{i=1}^{n} c_{i}^{2}=4 \sin ^{2} \theta
$$

or

(b)

$$
\begin{gathered}
\alpha_{i}=c_{i} t+d_{i}, \\
\alpha_{2 n+1}=2 t \cos \theta+\sum_{i=1}^{n} c_{i}\left(\frac{c_{n+i}}{2} t^{2}+d_{n+i} t\right)+c_{2 n+1},
\end{gathered}
$$

where $q=2 \cos \theta$ and $c_{i}(i=1,2, \ldots, 2 n+1), d_{i}(i=1,2, \ldots, 2 n)$ are arbitrary constants such that

$$
q^{2}+\sum_{i=1}^{2 n} c_{i}^{2}=4
$$

\section{REFEREN CES}

1. T. ADACHI: Curvature bound and trajectories for magnetic fields on a Hadamard surface. Tsukuba J. Math. 20 (1996), 225-230.

2. D. E. BlaIR: Riemannian geometry of contact and symplectic manifolds, Second edition. Birkhauser Boston, Inc., Boston, MA, 2010.

3. M. Barros, A. Romero, J. L. Cabrerizo and M. Fernández: The Gauss-LandauHall problem on Riemannian surfaces. J. Math. Phys. 46 (2005), 11290515 pp. 
4. J. L. Cabrerizo, M. Fernandez and J. S. Gomez: On the existence of almost contact structure and the contact magnetic field. Acta Math. Hungar. 125 (2009), 191-199.

5. G. Calvaruso, M. I. Munteanu and A. Perrone: Killing magnetic curves in threedimensional almost paracontact manifolds. J. Math. Anal. Appl. 426 (2015), 423-439.

6. J. T. Сho, J. Inoguchi and J. E. LeE: On slant curves in Sasakian 3-manifolds. Bull. Austral. Math. Soc. 74 (2006), 359-367.

7. J. T. CHO and J. E. LEE: Slant curves in contact pseudo-Hermitian 3-manifolds. Bull. Austral. Math. Soc. 78 (2008), 383-396.

8. A. Comtet: On the Landau levels on the hyperbolic plane. Ann. Physics 173 (1987), 185-209.

9. S. L. Druţă-Romaniuc, J. Inoguchi, M. I. Munteanu and A. I. Nistor: Magnetic curves in Sasakian manifolds. J. Nonlinear Math. Phys. 22 (2015), 428-447.

10. S. L. Druţă-Romaniuc, J. Inoguchi, M. I. Munteanu and A. I. Nistor: Magnetic curves in cosymplectic manifolds. Rep. Math. Phys. 78 (2016), 33-48.

11. Ş. GüvEnÇ and C. ÖzGür: On slant magnetic curves in S-manifolds. J. Nonlinear Math. Phys. 26 (2019), 536-554.

12. Ş. GüVEnÇ: On pseudo-Hermitian biharmonic slant curves in Sasakian space forms endowed with the Tanaka-Webster connection. Bull. Iran. Math. Soc. 46 (2020), 207223.

13. J. Inoguchi and M. I. Munteanu: Periodic magnetic curves in Berger spheres. Tohoku Math. J. 69 (2017), 113-128.

14. M. Jleli, M. I. Munteanu and A. I. Nistor: Magnetic trajectories in an almost contact metric manifold $\mathbb{R}^{2 N+1}$. Results Math. 67 (2015), 125-134.

15. M. Jleli and M. I. Munteanu: Magnetic curves on flat para-Kahler manifolds. Turkish J. Math. 39 (2015), 963-969.

16. L. D. LAndau and E. M. Lifshitz: Course of theoretical physics. Vol. 1. Mechanics. Third edition. Pergamon Press, Oxford-New York-Toronto, Ont., 1976.

17. M. I. Munteanu and A. I. Nistor: The classification of Killing magnetic curves in $S^{2} \times R$. J. Geom. Phys. 62 (2012), 170-182.

18. M. I. Munteanu and A. I. Nistor: A note on magnetic curves on $S^{2 n+1}$. C. R. Math. Acad. Sci. Paris 352 (2014), 447-449.

19. M. I. Munteanu: The Landau-Hall problem on canal surfaces. J. Math. Anal. Appl. 414 (2014), 725-733.

20. C. ÖzGüR and Ş. GüvEnÇ: On some types of slant curves in contact pseudo-Hermitian 3-manifolds. Ann. Polon. Math. 104 (2012), 217-228.

21. C. ÖzGür: On magnetic curves in the 3-dimensional Heisenberg group. Proc. Inst. Math. Mech. Natl. Acad. Sci. Azerb. 43 (2017), 278-286.

22. N. TANAKA: On non-degenerate real hypersurfaces, graded Lie algebras and Cartan connections. Japan. J. Math. (N.S.) 2 (1976), 131-190.

23. S. Tanno: Variational problems on contact Riemannian manifolds. Trans. Amer. Math. Soc. 314 (1989), 349-379.

24. S. M. Webster: Pseudo-Hermitian structures on a real hypersurface. J. Differential Geom. 13 (1978), 25-41. 
Şaban Güvenç

Faculty of Arts and Sciences

Department of Mathematics

Campus of Çă̆ış

10145 Balikesir, Turkey

sguvenc@balikesir.edu.tr

Cihan Özgür

Faculty of Arts and Sciences

Department of Mathematics

Campus of Çă̆ış

10145 Balikesir, Turkey

cozgur@balikesir.edu.tr 